\title{
In situ Thermal Shock of Lunar and Planetary Materials Using A Newly Developed MEMS Heating Holder in A STEM/SEM
}

Jane Y. Howe ${ }^{1}$, Michelle S. Thompson ${ }^{2}$, Stas Dogel ${ }^{3}$, Kota Ueda ${ }^{4}$, Tsuyoshi Matsumoto ${ }^{4}$, Hideki Kikuchi ${ }^{4}$, Matthew Reynolds ${ }^{5}$, Hooman Hosseinkhannazer ${ }^{5}$, and Thomas J. Zega ${ }^{6}$

1. Hitachi High-Technologies America Inc., Clarksburg, USA.

2. NASA Johnson Space Center, Houston, USA.

3. Hitachi High-Technologies Canada Inc., Toronto, Canada.

4. Hitachi High-Technologies Co., Hitachinaka, Japan.

5. Norcada Inc., Edmonton, Canada.

${ }^{6 .}$ University of Arizona, Tucson, USA.

The US Department of Energy's Basic Energy Sciences Office published a report entitled, "Future Electron Scattering and Diffraction" in 2014. In the report, it listed the "Lab-in-gap" dynamic microscope as one of the major instrumentation needs for enabling breakthrough scientific opportunities. Specifically, it called for sample stage and holder designs that would allow advanced in situ analyses [1]. We have recently manufactured a MEMS-based heating and electric bias holder that fits into the sample stage of the newly developed 200-kV Hitachi HF5000 transmission electron microscope. The new sample stage enables a wider, jewel-less sample holder design, providing a larger platform which is advantageous for laying out electric contacts and transportation of stimuli. As shown in Fig. 1a, the Hitachi "Blaze" Heating Holder comes with the MEMS-based heating chips, which are SiN membranes sandwiching a heating element, manufactured by Norcada Inc. The contact pad to the heating chip is a replaceable. Simulated temperature profiles at 850 and $1100{ }^{\circ} \mathrm{C}$ indicated the central $160 \mu \mathrm{m}$-diameter is isothermal (Figs.1b and 1c). At the center of the isothermal region, there are 19 sample wells in 5 arrays. These sample wells have a $30 \mathrm{~nm}$-thick SiN support film, providing electron transparency and exceptional chemical, thermal and mechanical stability.

In this paper, we report the in situ heating study of lunar and planetary materials using the Blaze heating holder [2]. The lunar soil samples were acquired from the Apollo 14 and 17 missions. To prepare the samples for in situ heating, we suspended particles in methanol and then transferred them onto the heating chip using a micro pipette. We analyzed the samples after the methanol had completely evaporated. Part of the heating study was carried out using the $300 \mathrm{kV}$ Hitachi HF3300 TEM/STEM located at the University of Toronto. Complementary work is also being pursued with in-depth heating studies in the probe-corrected $200 \mathrm{kV}$ Hitachi HF5000 TEM/STEM at the University of Arizona. Both microscopes are equipped with a secondary electron (SE) detector, which allow simultaneous secondary and transmitted election imaging to obtain the information from the surface (SEM) and bulk (annular dark field (ADF) and bright field (BF) of the lunar soil particles. Figure 2 documents the structural evolution of one particle before and after a total of eight thermal shocks with 1-sec duration over a temperature range from 20 to $940{ }^{\circ} \mathrm{C}$. Iron nanocrystals developed on the surface of the grain after the first thermal shock and continued to increase in size with each subsequent heat treatment. EELS analysis (not shown) further confirms the metallic nature of these iron nanocrystals.

In summary, the Blaze heating holder is proven to have enabled simulation of heating events occurred on airless bodies such as lunar and planetary materials. It can also be used to address questions on thermal annealing of ceramics and nanostructured materials [3]. 


\section{References:}

[1] E Hall et al, Report of the Basic Energy Sciences Workshop on Future of Electron Scattering and Diffraction (2014) p. 28.

[2] MS Thompson, TJ Zega, and JY Howe, Meteoritics \& Planetary Science 52 (2017) on line.

[3] Research supported by National Science Foundation grant number 1531243 and NASA grant numbers NNX15AJ22G and NNX12AL47G. Microscopic studies were carried out at the Ontario Centre for the Characterisation of Advanced Materials (OCCAM), University of Toronto, and the Kuiper Core Imaging and Microscopy Facility, University of Arizona.

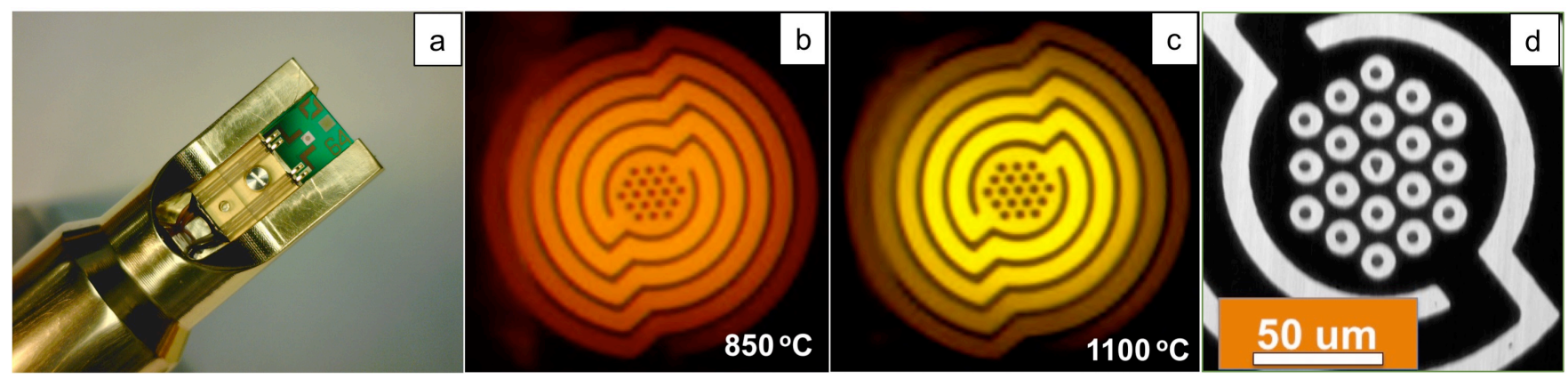

Figure 1. Blaze heating holder and the MEMS-based heating chip fitted for the Hitachi HF5000 TEM/STEM (a); the simulated temperature profiles for $850(\mathrm{~b})$ and $1100{ }^{\circ} \mathrm{C} \mathrm{(c)}$; and the 19 sample wells at the isothermal center $(d)$.
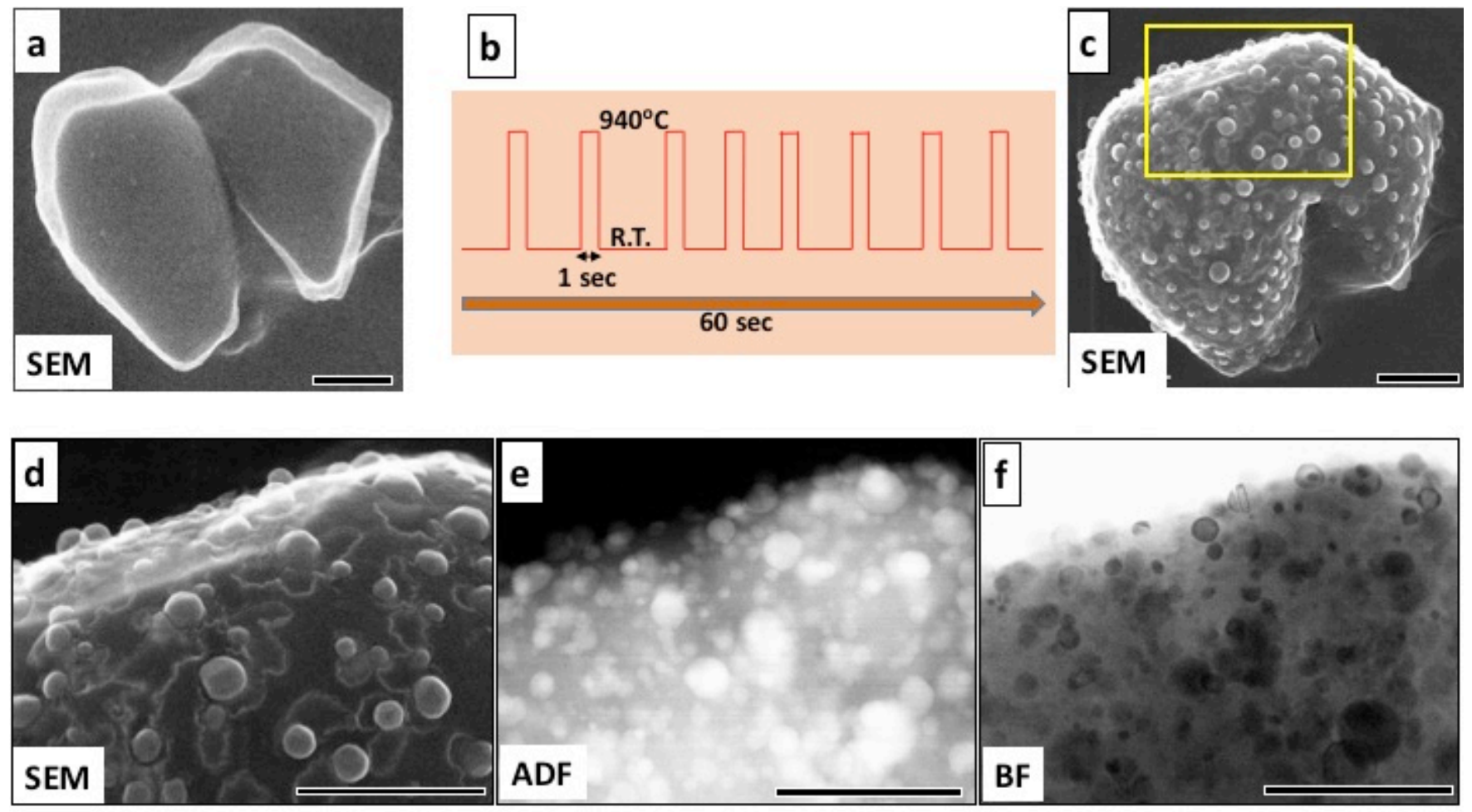

Figure 2. A lunar soil particle before (a) and after a total of 8 thermal shock events (c to f). The heating schedule is illustrated in (b). The simultaneous SE/ADF/BF imaging of the area in yellow frame of (c) (all scale bars are $200 \mathrm{~nm}$ ). 